\title{
Light-Harvesting Nanotubes Formed by Supramolecular Assembly of Aromatic Oligophosphates**
}

\author{
Caroline D. Bösch, Simon M. Langenegger and Robert Häner*
}

\begin{abstract}
A 2,7-disubstituted phosphodiester-linked phenanthrene trimer forms tubular structures in aqueous medium. Chromophores are arranged in H-aggregates. Incorporation of small quantities of pyrene results in the development of light-harvesting nanotubes, in which phenanthrenes act as antenna chromophores and pyrenes as energy acceptors. The process of energy collection is most efficient after excitation at the phenanthrene H-band. Fluorescence quantum yields up to $23 \%$ are reached in pyrene doped, supramolecular nanotubes.
\end{abstract}

The construction of artificial light-harvesting complexes has received much attention in recent years. ${ }^{[1-11]}$ In light-harvesting systems, energy is absorbed by numerous chromophores and transferred to an acceptor. Polymers ${ }^{[12-19]}$ and dendrimers ${ }^{[20-26]}$ were used to arrange multiple organic chromophores around acceptor molecules. Efficient transport of the excitation energy is a key aspect for the construction of effective light-harvesting systems. ${ }^{[27]}$ The degree of structural order determines the level of electronic coupling and, thus, the efficiency of energy transfer in chromophore arrays. ${ }^{[2,3,28,29]}$ One way of assembling highly organized molecular arrays is provided by supramolecular polymerization. ${ }^{[30-36]}$ Inspired by the unique electronic properties of carbon nanotubes, ${ }^{[37,38]}$ supramolecular organic nanotubes appear as particularly attractive scaffolds for the molecular organization of chromophore aggregates. Due to the non-covalent nature of interaction, supramolecular polymers offer a high degree of modularity and, hence, flexibility for their construction and potential functionalisation. The assembly of supramolecular nanotubes with light-harvesting properties has been shown for amphiphilic monomers ${ }^{[39-41]}$ and aromatic peptides. ${ }^{[42]}$ In previous work we described the formation of 1dimensional light-harvesting supramolecular polymers from short aromatic oligophosphates. ${ }^{[43]}$ The formation of 2-dimensional (2D) polymers would allow extending considerably the number of structurally organized, energy collecting chromophores. The assembly of 2D polymers was demonstrated with 1,6- and 2,7linked pyrene oligomers. ${ }^{[4-46]}$ Here, we describe the formation of light-harvesting supramolecular nanotubes from 2,7-linked oligophenanthrenes doped with pyrene acceptor chromophores.

Oligomers 1 and 2 (Figure 1) were assembled on a phenanthrenederived solid support using phosphoramidite chemistry. The required phenanthrene and pyrene phosphoramidites were synthesized from 2,7-dibromophenanthrene, ${ }^{[47]}$ or the commercially available 2,7-dibromopyrene (see Supporting Information). Trimer 1 consists of three phenanthrene units joined by phosphodiester groups attached via butynol linkers in positions 2 and 7. The structure of $\mathbf{2}$ is similar to $\mathbf{1}$, except that the phenanthrene in the central position has been replaced by a 2,7-substituted pyrene.
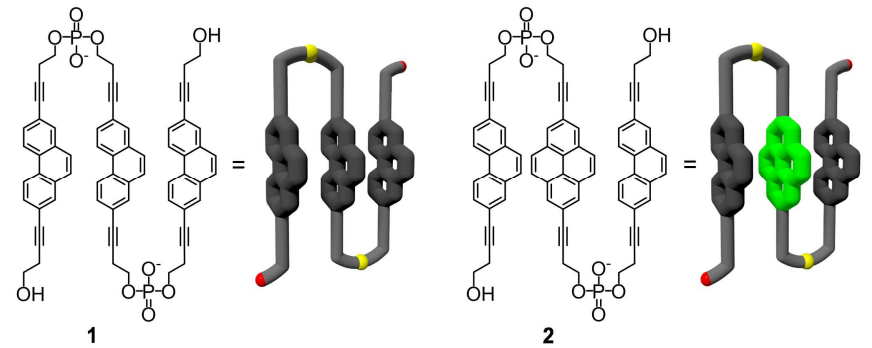

Figure 1. Chemical structure and illustrative model representations of trimers 1 and 2. Pyrene is highlighted green.

The effect of solvent polarity on the aggregation behavior of $\mathbf{1}$ is shown by changes in the absorption spectra (Figure 2). In ethanol, the oligomer is well soluble and the spectrum closely resembles that of the phenanthrene monomer (Supporting Information). In more polar, aqueous environment (see Figure 2 for experimental details), phenanthrenes exhibit strong aggregation behavior as revealed by a substantial hypochromism, especially at 270 and $316 \mathrm{~nm}$, and the characteristic changes in the vibronic structure. ${ }^{[48-53]}$ Phenanthrene aggregation leads to the disappearance of the $270 \mathrm{~nm}$ band and occurrence of an H-band $(243 \mathrm{~nm}) \cdot{ }^{[48,54]}$ This suggests that oligomer 1 forms supramolecular polymers in which the phenanthrenes are arranged in extended $\mathrm{H}$-aggregates. Heating of the aqueous solution to $80^{\circ} \mathrm{C}$ (red curve) leads to disassembly of the polymers and concomitant disappearance of the H-band.

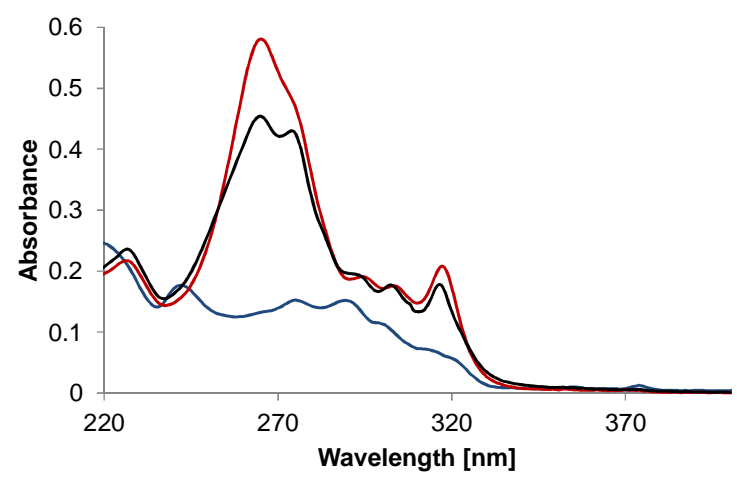

Figure 2. Absorption spectra of 1 in $10 \mathrm{mM}$ sodium phosphate buffer $\mathrm{pH} 7.0$, $10 \%$ ethanol (blue: at $20^{\circ} \mathrm{C}$, red: at $80^{\circ} \mathrm{C}$ ) and in ethanol at $20^{\circ} \mathrm{C}$ (black), concentration: $1 \mu \mathrm{M}$.

Trimer 1 forms tubular structures in aqueous solution. Atomic force microscopy (AFM, Figure 3) reveals elongated objects reaching a length of several micrometers. The measured height of the polymers after deposition on $\mathrm{NiCl}_{2}$-treated mica ${ }^{[55]}$ is in the range of 4-4.5 nm. This corresponds to a double-layer of $\pi$-stacked phenanthrenes 
formed by the deposition of supramolecular tubes on the surface. ${ }^{[46]}$ The diameter of the nanotubes varies in the range of 50-150 nm (Supporting Information). The tubular morphology of the nanostructures was confirmed by transmission electron microscopy (TEM) experiments (Figure 3). Notably, polymerization of oligomer 1 exclusively results in the formation of tubular objects. This is in contrast to findings with trimers of the iso-structural 2,7-pyrene, ${ }^{[46]}$ which leads to the simultaneous formation of nanosheets and nanotubes.
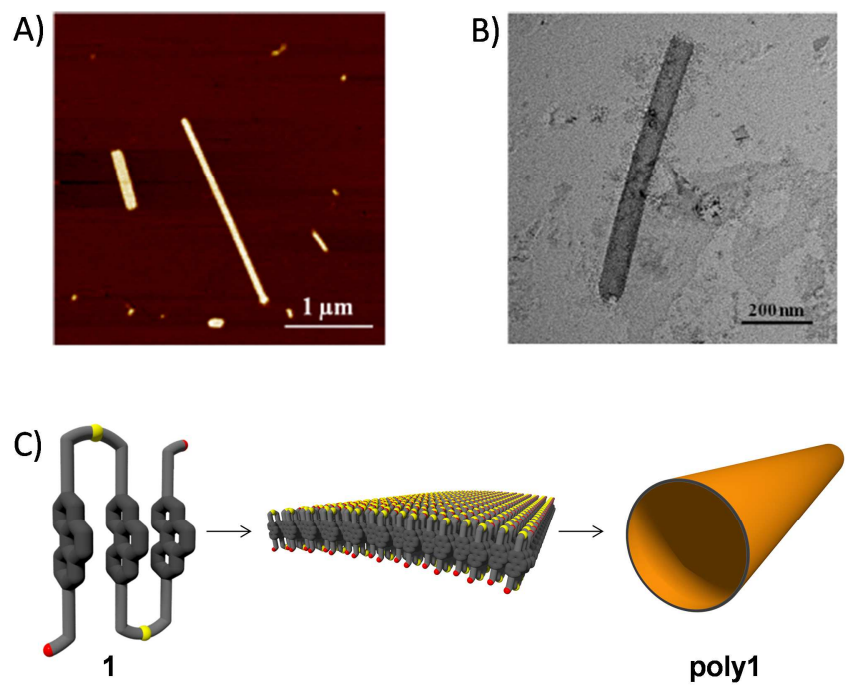

Figure 3. A) AFM image of supramolecular polymers (poly1) assembled in aqueous solution and deposited on mica using $\mathrm{NiCl}_{2}$ as surface binding agent. Formation of the nanotubes is accomplished by slow cooling of a $1 \mu \mathrm{M}$ solution of trimer 1 from $80^{\circ} \mathrm{C}$ to room temperature in water $(10 \mathrm{mM}$ sodium phosphate buffer, $\mathrm{pH} 7.0$ ) containing $10 \% \mathrm{v} / \mathrm{v}$ of ethanol. B) TEM image of a phenanthrene nanotube. C) Illustration of the self-assembly of phenanthrene oligomers 1 . Hydrophobic phenanthrene units are arranged in a sheet-like manner with hydroxyl-groups (red) and phosphates (yellow) located on its outsides.

The fluorescence spectrum of poly1 measured in aqueous medium shows a structured emission band with maxima at 371 and $391 \mathrm{~nm}$ when excited at $316 \mathrm{~nm}$ (Figure 4). Excitation at $243 \mathrm{~nm}$ (H-band) leads to changes in the shape of the emission band. The maxima are shifted by $5 \mathrm{~nm}$ to the red ( 376 and $396 \mathrm{~nm}$ ) and a broad band newly appears around $425 \mathrm{~nm}$, which most likely arises from excimer formation. ${ }^{[56]}$ The wavelength dependence of phenanthrene fluorescence has been reported previously and was attributed to solvation effects. ${ }^{[57]}$ The fluorescence quantum yield $(\Phi)$ also depends significantly on the excitation wavelength. For excitation at $316 \mathrm{~nm}$, a value of $7 \%$ was obtained (see Supporting Information for details), whereas excitation at $243 \mathrm{~nm}$ (H-band) renders a value of only $3 \%$. A red-shifted fluorescence and a low quantum yield are well-known features of $\mathrm{H}$-aggregates ${ }^{[58]}$ Excitation spectra (Figure 5) further demonstrate the strong dependence of fluorescence on the excitation wavelength. The spectrum obtained by recording the emission at $391 \mathrm{~nm}$ resembles the absorption spectrum of oligomer 1 dissolved in ethanol. Monitoring of the emission at $412 \mathrm{~nm}$ gives a spectrum with a peak at $243 \mathrm{~nm}$, which coincides with the H-band in the absorption spectrum of poly1. Finally, the excitation spectrum recorded at $437 \mathrm{~nm}$ is comparable to the absorption spectrum of poly1 obtained in aqueous medium exhibiting again the characteristic band at $243 \mathrm{~nm}$ and a comparatively reduced intensity at 275 and $316 \mathrm{~nm}$. Thus, H-aggregation leads to a red-shifted fluorescence.

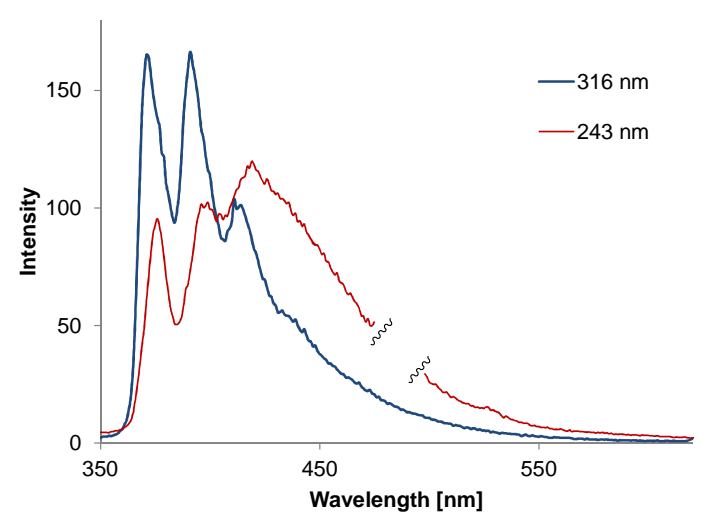

Figure 4. Fluorescence spectrum of poly1. Conditions: $1 \mu \mathrm{M}$ in $10 \mathrm{mM}$ sodium phosphate buffer $\mathrm{pH} 7.0,10 \%$ ethanol; $\lambda_{\mathrm{ex}} 316 \mathrm{~nm}$ (blue) and $243 \mathrm{~nm}$ (red); excitation slit: $5 \mathrm{~nm}$, emission slit: $5 \mathrm{~nm}$.

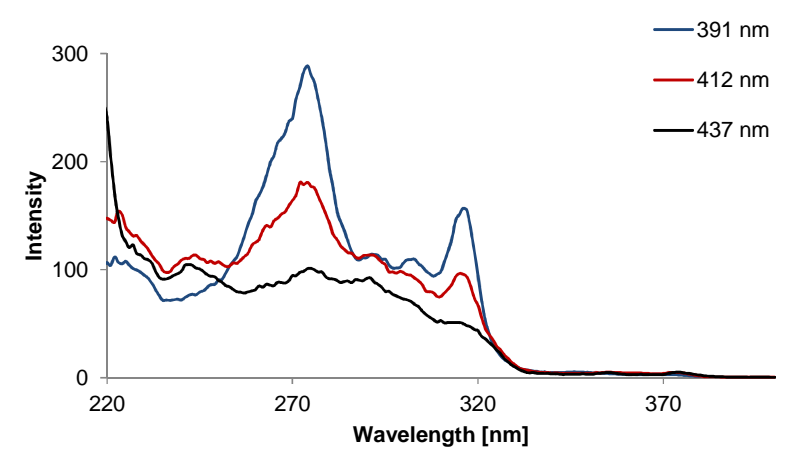

Figure 5. Excitation spectra of poly1. Conditions: $1 \mu \mathrm{M}$ in $10 \mathrm{mM}$ sodium phosphate buffer pH 7.0, 10\% ethanol; $\lambda_{\mathrm{em}} 391 \mathrm{~nm}$ (blue), $412 \mathrm{~nm}$ (red) and 437 $\mathrm{nm}$ (black); excitation slit: $5 \mathrm{~nm}$, emission slit: $5 \mathrm{~nm}$.

We previously observed efficient energy transfer from 1,8disubstituted phenanthrenes to acceptor pyrenes in light-harvesting supramolecular polymers fibers. ${ }^{[43]}$ To test if the present nanotubes formed of 2,7-dialkynyl phenanthrenes also possess light-harvesting properties, polymerization of $\mathbf{1}$ was carried out in the presence of small quantities of the pyrene containing oligomer 2 . This leads to the formation of pyrene-doped supramolecular nanotubes, as illustrated in Figure 6.

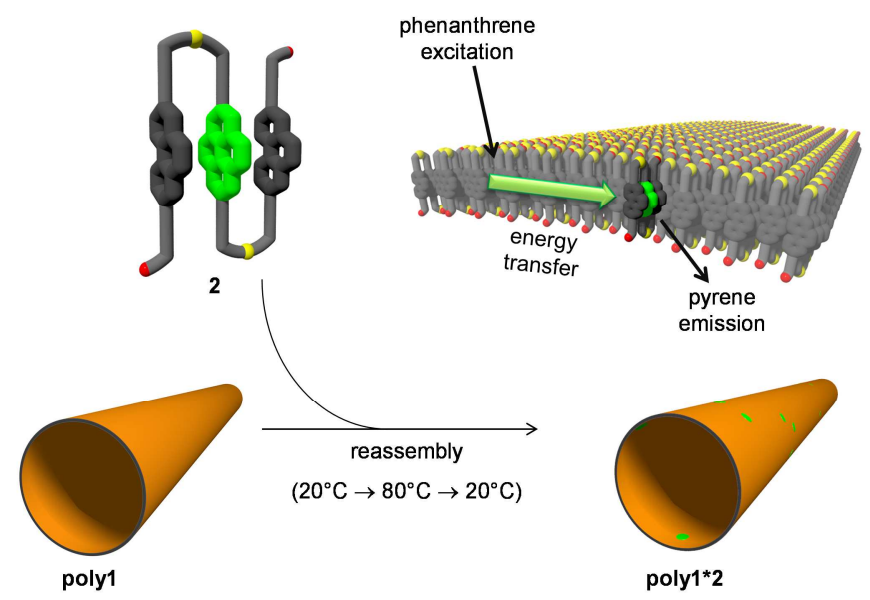

Figure 6. Illustration of light-harvesting nanotubes: supramolecular polymerization of aromatic oligophosphates leads to the formation of pyrenedoped phenanthrene nanotubes. Excitation of phenanthrene (grey) is followed by energy transfer to pyrene acceptor molecules (green). 
A comparison of the fluorescence spectra of nanotubes consisting exclusively of phenanthrenes and nanotubes containing $1.2 \%$ of pyrene molecules is displayed in Figure 7. Already small quantities of pyrene acceptor lead to a substantial increase in the fluorescence intensity upon irradiation at $243 \mathrm{~nm}$, which corresponds to the $\mathrm{H}$ band in the spectrum of the phenanthrene polymer. The effect of increasing pyrene content in the nanotubes on the intensity of pyrene fluorescence is displayed in Figure 8. The maximum intensity is reached with a pyrene content $\sim 7 \%$. This confirms that the supramolecular nanotube functions as a light-harvesting antenna in which excitation energy is transferred from phenanthrenes to pyrene acceptor atoms. Phenanthrene excitation at $316 \mathrm{~nm}$ also leads to a steady increase of pyrene emission, although not as much as excitation at the H-band (Supporting Information). The ratio of pyrene/phenanthrene emission is higher if the antenna is excited at $243 \mathrm{~nm}$. This is in agreement with the theory that the increased radiative lifetime for the relaxed excited state in $\mathrm{H}$-aggregates is beneficial for efficient energy transfer. ${ }^{[59]}$ The quantum yield of the light-harvesting antenna containing $7.3 \%$ pyrene, however, is the same $(23 \%)$ for both of these excitation wavelengths.

It is important to note that incorporation of oligomer $\mathbf{2}$ into the nanotubes takes place only if the polymerization process (heating of the oligomer solution to $80{ }^{\circ} \mathrm{C}$ and then cooling to $20^{\circ} \mathrm{C}$ ) is carried out in the presence of $\mathbf{2}$. No incorporation is observed upon addition of $\mathbf{2}$ to preformed nanotubes poly1 at ambient temperature. Furthermore, oligomer $\mathbf{2}$ alone does not exhibit light-harvesting properties, which is demonstrated by titration experiments carried out with solutions not containing oligomer 1 (Supporting Information). Finally, the presence of small quantities of oligomer 2 has no effect on the morphology of the supramolecular polymers. AFM samples that were prepared using a solution containing 0.5

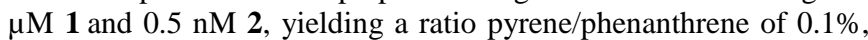
revealed only objects of identical shape as those formed of oligomer $\mathbf{1}$ alone (Supporting Information).

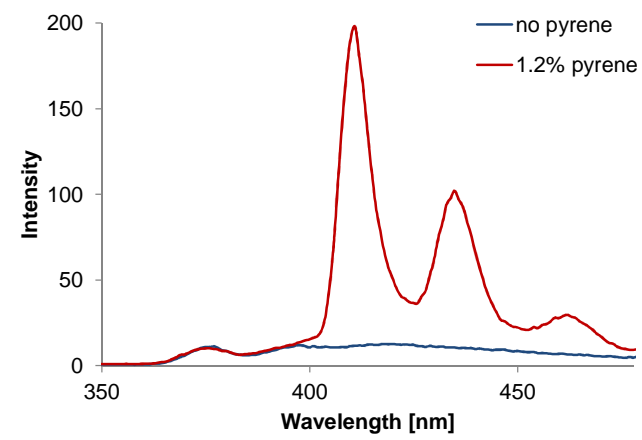

Figure 7. Fluorescence spectra of assembled phenanthrene nanotubes in the absence (black) and in the presence of $1.2 \%$ of pyrene (red). Concentration of 1 $=0.5 \mu \mathrm{M}$ in $10 \mathrm{mM}$ sodium phosphate buffer $\mathrm{pH} 7.0,10 \%$ ethanol. $\lambda_{\mathrm{ex}} 243 \mathrm{~nm}$; excitation slit: $2.5 \mathrm{~nm}$, emission slit: $5 \mathrm{~nm}$.

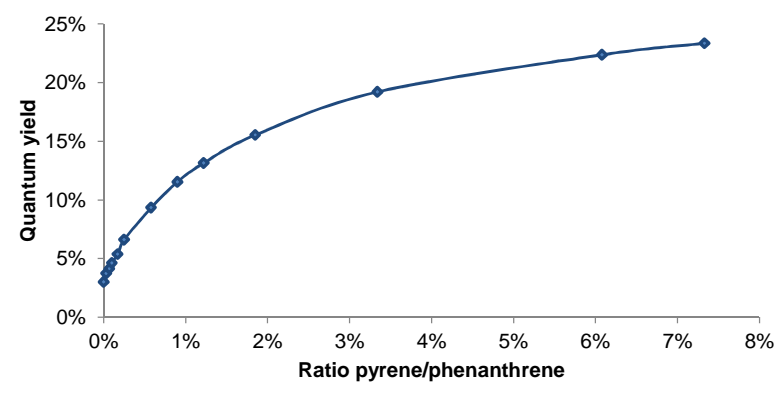

Figure 8. Quantum yield of light-harvesting supramolecular nanotubes as a function of the pyrene/phenanthrene ratio. Conditions as in Figure 7.
In conclusion, the self-assembly of aromatic oligomers into nanotubes with light-harvesting properties was demonstrated. Phosphodiester-linked phenanthrene trimers form H-aggregated, supramolecular polymers in aqueous medium. AFM and TEM imaging reveals tubular objects with a length of up to several micrometers. Nanotubes assembled entirely from phenanthrenes exhibit weak fluorescence. Incorporation of small quantities of pyrene units, however, leads to a strong increase of fluorescence. The supramolecular nanotubes function as light-harvesting antennae in which excitation energy is transferred from phenanthrenes to pyrene acceptor atoms. The observed antenna effect is highest when the nanotubes are excited at the phenanthrene H-band. Fluorescence quantum yields are reaching $23 \%$ for nanotubes containing $7 \%$ pyrene.

Received: ((will be filled in by the editorial staff)) Published online on ((will be filled in by the editorial staff $)$ )

Keywords: Energy transfer $\cdot \mathrm{H}$-aggregate $\bullet$ supramolecular polymers - light-harvesting antenna $\cdot$ nanotube

[1] D. Gust, T. A. Moore, A. L. Moore, Acc.Chem.Res. 2001, 34, 40-48.

[2] M. R. Wasielewski, Acc.Chem.Res. 2009, 42, 1910-1921.

[3] G. D. Scholes, G. R. Fleming, A. Olaya-Castro, R. van Grondelle, Nat.Chem. 2011, 3, 763-774.

[4] I. McConnell, G. H. Li, G. W. Brudvig, Chem.Biol. 2010, 17, 434-447.

[5] N. Aratani, D. Kim, A. Osuka, Acc.Chem.Res. 2009, 42, 1922-1934.

[6] W. Su, V. Bonnard, G. A. Burley, Chem.Eur.J. 2011, 17, 7982-7991.

[7] K. Rao, V, K. Datta, M. Eswaramoorthy, S. J. George, Chem.Eur.J. 2012, 18, 2184-2194.

[8] R. Ziessel, A. Harriman, Chem.Commun. 2011, 47, 611-631.

[9] D. Holten, D. F. Bocian, J. S. Lindsey, Acc.Chem.Res. 2002, 35, 57-69.

[10] M. S. Choi, T. Yamazaki, I. Yamazaki, T. Aida, Angew.Chem.Int.Ed. 2004, 43, 150-158.

[11] H. Imahori, J.Phys.Chem.B 2004, 108, 6130-6143,

[12] S. E. Webber, Chem.Rev. 1990, 90, 1469-1482.

[13] D. Ng, J. E. Guillet, Macromol. 1982, 15, 724-727.

[14] V. S. Lin, S. G. DiMagno, M. J. Therien, Science 1994, 264, 11051111

[15] R. W. Wagner, J. S. Lindsey, J.Am.Chem.Soc. 1994, 116, 9759-9760.

[16] M. A. Fox, Acc.Chem.Res. 1992, 25, 569-574.

[17] D. M. Watkins, M. A. Fox, J.Am.Chem.Soc. 1994, 116, 6441-6442.

[18] I. Hwang, G. D. Scholes, Chem.Mater. 2011, 23, 610-620.

[19] E. Collini, G. D. Scholes, Science 2009, 323, 369-373.

[20] V. Balzani, A. Credi, M. Venturi, Chemsuschem 2008, 1, 26-58.

[21] C. Devadoss, P. Bharathi, J. S. Moore, J.Am.Chem.Soc. 1996, 118 , 9635-9644.

[22] S. L. Gilat, A. Adronov, J. M. J. Fréchet, Angew.Chem.Int.Ed. 1999, $38,1422-1427$.

[23] A. Adronov, J. M. J. Fréchet, Chem.Commun. 2000, 1701-1710.

[24] J. F. Galindo, E. Atas, A. Altan, D. G. Kuroda, S. Fernandez-Alberti, S. Tretiak, A. E. Roitberg, V. D. Kleiman, J.Am.Chem.Soc. 2015, 137, 11637-11644.

[25] C. Giansante, P. Ceroni, V. Balzani, F. Voegtle, Angew.Chem.Int.Ed. 2008, 47, 5422-5425.

[26] H. Lee, Y. H. Jeong, J. H. Kim, I. Kim, E. Lee, W. D. Jang, J.Am.Chem.Soc. 2015, 137, 12394-12399.

[27] A. N. Melkozernov, J. Barber, R. E. Blankenship, Biochemistry 2006, 45, 331-345.

[28] Z. G. Fetisova, A. M. Freiberg, K. E. Timpmann, Nature 1988, 334, 633-634.

[29] N. Sakai, S. Matile, Beilstein J.Org.Chem. 2012, 8, 897-904

[30] T. F. A. de Greef, M. M. J. Smulders, M. Wolffs, A. P. H. J. Schenning, R. P. Sijbesma, E. W. Meijer, Chem.Rev. 2009, 109, 56875754 .

[31] F. J. M. Hoeben, P. Jonkheijm, E. W. Meijer, A. P. H. J. Schenning, Chem.Rev. 2005, 105, 1491-1546.

[32] H. Cui, M. J. Webber, S. I. Stupp, Biopolymers 2010, 94, 1-18. 


\section{Angewandte}

\section{Communications}

[33] T. Aida, E. Meijer, S. I. Stupp, Science 2012, 335, 813-817.

[34] D. Görl, X. Zhang, F. Würthner, Angew.Chem.Int.Ed. 2012, 51, 63286348.

[35] M. J. Webber, E. A. Appel, E. W. Meijer, R. Langer, Nat.Mater. 2016 15, 13-26.

[36] E. Krieg, M. M. C. Bastings, P. Besenius, B. Rybtchinski, Chem.Rev. 2016, DOI: 10.1021/acs.chemrev.5b00369.

[37] R. H. Baughman, A. A. Zakhidov, W. A. de Heer, Science 2002, 297, 787-792.

[38] X. Zang, Q. Zhou, J. Chang, Y. Liu, L. Lin, Microelectron.Eng. 2015 , 132, 192-206.

[39] N. Kameta, K. Ishikawa, M. Masuda, M. Asakawa, T. Shimizu, Chem.Mater. 2012, 24, 209-214.

[40] D. Eisele, C. Cone, E. Bloemsma, S. Vlaming, C. van der Kwaak, R. Silbey, M. Bawendi, J. Knoester, J. Rabe, D. Vanden Bout, Nat.Chem. 2012, 4, 655-662.

[41] H. Shao, J. Seifert, N. C. Romano, M. Gao, J. J. Helmus, C. P. Jaroniec, D. A. Modarelli, J. R. Parquette, Angew.Chem.Int.Ed. 2010, 49, 7688-7691.

[42] J. H. Kim, M. Lee, J. S. Lee, C. B. Park, Angew.Chem.Int.Ed. 2012 $51,517-520$

[43] C. B. Winiger, S. Li, G. R. Kumar, S. M. Langenegger, R. Häner, Angew.Chem.Int.Ed. 2014, 53, 13609-13613.

[44] M. Vybornyi, A. V. Rudnev, S. M. Langenegger, T. Wandlowski, G. Calzaferri, R. Häner, Angew.Chem.Int.Ed. 2013, 52, 11488-11493.

[45] M. Vybornyi, A. Rudnev, R. Häner, Chem.Mater. 2015, 27, 14261431.

[46] M. Vybornyi, Y. B.-C. Hechevarria, M. Glauser, A. V. Rudnev, R. Häner, Chem. Commun. 2015, 51, 16191-16193.

[47] J. W. Ciszek, J. M. Tour, Tetrahedron Lett. 2004, 45, 2801-2803.

[48] F. C. Spano, Acc.Chem.Res. 2010, 43, 429-439.

[49] A. E. Clark, C. Y. Qin, A. D. Q. Li, J.Am.Chem.Soc. 2007, 129, 75867595.

[50] H. Bittermann, D. Siegemund, V. L. Malinovskii, R. Häner, J.Am.Chem.Soc. 2008, 130, 15285-15287.

[51] M. Hariharan, K. Siegmund, Y. Zheng, H. Long, G. C. Schatz, F. D. Lewis, J.Phys.Chem.C 2010, 114, 20466-20471.

[52] C. B. Winiger, S. M. Langenegger, G. Calzaferri, R. Häner, Angew.Chem.Int.Ed. 2015, 54, 3643-3647.

[53] P. S. Rukin, A. Y. Freidzon, A. V. Scherbinin, V. A. Sazhnikov, A. A Bagaturyants, M. V. Alfimov, PhysChemChemPhys 2015, 17, 1699717006.

[54] A. Eisfeld, J. S. Briggs, Chem.Phys. 2006, 324, 376-384.

[55] A. V. Rudnev, V. L. Malinovskii, A. L. Nussbaumer, A. Mishchenko, R. Häner, T. Wandlowski, Macromol. 2012, 45, 5986-5992.

[56] J. B. Birks, Rep.Prog.Phys. 1975, 38, 903-974.

[57] E. Lendvay, I. Hornyak, J.Luminescence 1974, 9, 18-31.

[58] M. Sauer, J. Hofkens, J. Enderlein, Basic Principles of Fluorescence Spectroscopy, Wiley-VCH Verlag GmbH \& Co, 2011.

[59] A. T. Haedler, K. Kreger, A. Issac, B. Wittmann, M. Kivala, N. Hammer, J. Kohler, H. W. Schmidt, R. Hildner, Nature 2015, 523 , 196-199. 


\section{Angewandte}

\section{Communications}

Entry for the Table of Contents

\section{Light-Harvesting Nanotubes}

Caroline D. Bösch, Simon M.

Langenegger and Robert Häner*

$$
\text { Page - Page }
$$

Light-Harvesting Nanotubes Formed by Supramolecular Assembly of Aromatic Oligophosphates
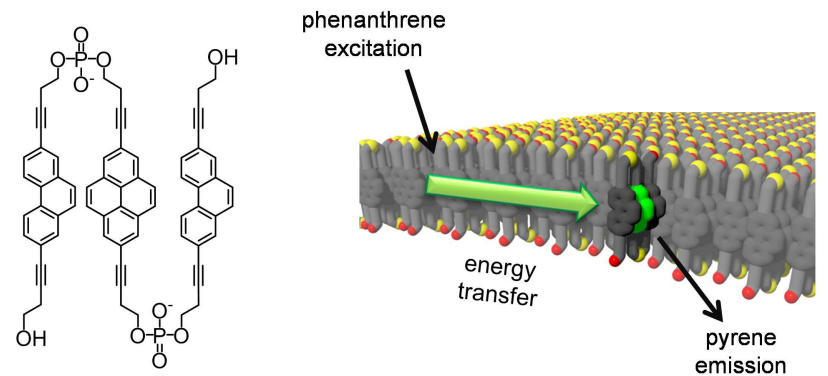

Phosphodiester-linked, 2,7-disubstituted phenanthrene trimers form supramolecular nanotubes in aqueous medium. Phenanthrenes are arranged in $\mathrm{H}$-aggregates. Incorporation of small amounts of pyrene acceptor molecules leads to highly efficient light-harvesting nanotubes.

[*] C.D. Bösch, Dr. S.M. Langenegger and Prof. Dr. R. Häner* Department of Chemistry and Biochemistry University of Bern

Freiestrasse 3, 3012 Bern, Switzerland

E-mail: robert.haener@dcb.unibe.ch Homepage: http://haener.dcb.unibe.ch

[**] Financial support by the Swiss National Foundation (grant 200020_149148) and NCCR MUST is gratefully acknowledged.

Supporting information for this article is available on the WWW under http://dx.doi.org/10.1002/anie.201xxxxxx. 